\title{
Intersubunit Interactions in EAAT4 Glutamate Transporters
}

\author{
Delany Torres-Salazar ${ }^{1,2}$ and Christoph Fahlke ${ }^{1,2,3,4}$ \\ ${ }^{1}$ Abteilung Neurophysiologie, Medizinische Hochschule, 30625 Hannover, Germany, ${ }^{2}$ Abteilung Physiologie, Rheinisch-Westfälische Technische \\ Hochschule Aachen, 52074 Aachen, Germany, ${ }^{3}$ Centro de Estudios Cientificos, Valdivia 509000, Chile, and ${ }^{4}$ Zentrum für Systemische Neurowissenschaften, \\ 30559 Hannover, Germany
}

Excitatory amino acid transporters (EAATs) play a central role in the termination of synaptic transmission and in extracellular glutamate homeostasis in the mammalian CNS. A functional transporter is assembled as oligomer consisting of three subunits, each of which appears to transport glutamate independently from the neighboring subunits. EAATs do not only sustain a secondary-active glutamate transport but also function as anion channel. We here address the question whether intersubunit interactions play a role in poremediated anion conduction. We expressed a neuronal isoform, EAAT4, heterologously in Xenopus oocytes and mammalian cells and measured glutamate flux and anion currents under various concentrations of $\mathrm{Na}^{+}$and glutamate. EAAT4 anion channels are active in the absence of both substrates, and increasing concentrations activate EAAT4 anion currents with a sigmoidal concentration dependence. Because only one glutamate molecule is cotransported per uptake cycle, the cooperativity between glutamate binding sites most likely arises from an interaction between different carrier domains. This interaction is modified by two point mutations close to the putative glutamate binding site, G464S and Q467S. Both mutations alter the dissociation constants and Hill coefficient of the substrate dependence of anion currents, leaving the concentration dependence of glutamate uptake unaffected. Our results demonstrate that glutamate carriers cooperatively interact during anion channel activation.

Key words: glutamate transporter; chloride channel; synaptic transmission; patch clamp; neurotransmitter; cell excitability

\section{Introduction}

Excitatory amino acid transporters (EAATs) sustain two fundamentally distinct transport mechanisms. They function as cotransporters of glutamate, sodium, potassium, and protons ions ("coupled transport") (Zerangue and Kavanaugh, 1996; Levy et al., 1998) and as anion channels ("uncoupled transport") (Fairman et al., 1995; Wadiche et al., 1995; Billups et al., 1996; Larsson et al., 1996; Melzer et al., 2003). Separate EAAT isoforms exhibit different anion selectivities (Melzer et al., 2003), and mutations in EAAT3 affect anion permeability ratios of the associated anion currents (Ryan et al., 2004). The EAAT protein itself is therefore thought to form the underlying anion-selective ion conduction pathway.

The coupled glutamate transport ensures low resting extracellular glutamate levels and prevents neuronal damage by excessive glutamate receptor activation in the mammalian CNS. EAATassociated anion currents were suggested to support electrogenic glutamate uptake by clamping the membrane potential to negative values (Wadiche et al., 1995). Additionally, EAAT anion channels might play a direct role in electric signaling of certain excitable cells (Amara and Fontana, 2002; Melzer et al., 2005).

The high-resolution structure of a bacterial glutamate trans-

Received 0ct. 24, 2005; revised May 9, 2006; accepted June 8, 2006.

These studies were supported by Deutsche Forschungsgemeinschaft FOR450 (C.F.). We thank Dr. J. Rothstein for providing the expression construct for rEAAT4, Dr. Patricia Hidalgo, Nico Melzer, and Doreen Nothmann for helpful discussions, and Barbara Poser for excellent technical assistance.

Correspondence should be addressed to Christoph Fahlke, Abteilung Neurophysiologie, Medizinische Hochschule Hannover, Carl-Neuberg-Strasse 1, D-30625 Hannover, Germany. E-mail: fahlke.christoph@mh-hannover.de. DOI:10.1523/JNEUROSCI.4545-05.2006

Copyright $\odot 2006$ Society for Neuroscience $\quad$ 0270-6474/06/267513-10\$15.00/0 porter (Yernool et al., 2004) and recent fluorescence resonance energy transfer analysis (Koch and Larsson, 2005) provided first insights into likely conformational changes underlying glutamate transport. In contrast, the molecular function of the EAATassociated anion channel is only poorly understood. EAAT glutamate transporters assemble as homotrimeric complexes (Haugeto et al., 1996; Gendreau et al., 2004; Yernool et al., 2004; Grewer et al., 2005; Koch and Larsson, 2005). Trimerization of monomers occurs efficiently during or shortly after synthesis of the individual subunits resulting in stable trimers as the sole native and functional oligomeric state (Gendreau et al., 2004). The trimeric structure thus mediates carrier- and pore-mediated transport processes. Although each subunit is thought to transport glutamate independently from the rest of the oligomer (Grewer et al., 2005; Koch and Larsson, 2005), it is presently unclear whether each subunit is also capable of anion transport by itself (Ryan et al., 2004; Grewer et al., 2005) or whether the EAAT anion pore is jointly formed by several subunits (Eskandari et al., 2000). We here study glutamate uptake and anion conduction at various concentrations of transporter substrates such as $\mathrm{Na}^{+}$and glutamate. Experiments were performed with wild-type (WT) EAAT4 as well as with mutant transporters bearing two point mutations, G464S and Q467S, that are located in the neighborhood of the glutamate binding pocket (Zhang and Kanner, 1999; Yernool et al., 2004). We demonstrate that several cooperatively interacting glutamate binding sites need to be occupied to activate the anion channel. G464S and Q467S alter the apparent dissociation constants, the binding cooperativity, and gating of the EAAT4 anion channels. Our results demonstrate that individual subunits interact in activating EAAT4 anion channels, 
fully consistent with the notion of anion channels that are either jointly formed by three subunits or consist of three cooperatively interacting protopores.

\section{Materials and Methods}

Expression of EAAT4 in mammalian cells. The pcDNA3.1-rEAAT4 construct was kindly provided by Dr. J. Rothstein (Johns Hopkins University, Baltimore, MD). Transient transfection of tsA201 cells using the $\mathrm{Ca}_{3}\left(\mathrm{PO}_{4}\right)_{2}$ technique was performed as described previously (Melzer et al., 2003). To identify cells with a high probability of expressing recombinant transporters, cells were cotransfected with a plasmid encoding the CD8 antigen and incubated $5 \mathrm{~min}$ before use with polystyrene microbeads precoated with anti-CD8 antibodies (Dynabeads M-450 CD 8; Dynal, Great Neck, NY). The CD8/EAAT4 cDNA ratio was adjusted to ensure that almost every cell with beads exhibited currents with the characteristic properties shown in Figure 1. Point mutations were introduced by PCR-based strategies. For each construct, two independent recombinants from the same transformation were examined and shown to exhibit indistinguishable functional properties. Cotransfection experiments with WT and R501C EAAT4 were performed at a 1:1 and a 1:4 molar ratio of WT and mutant EAAT4 CDNA.

Electrophysiology. Standard whole-cell patch-clamp recordings were performed using an Axopatch 200B (Molecular Devices, Palo Alto, CA) amplifier. Borosilicate pipettes were pulled with resistances of 1.0-2.2 $\mathrm{M} \Omega$. To reduce voltage errors attributable to uncompensated series resistances, we routinely compensated $>80 \%$ of the series resistance by an analog procedure and excluded cells with $>10 \mathrm{nA}$ maximum anion currents from the analysis. This procedure results in a calculated voltage error below $5 \mathrm{mV}$ in all analyzed cells under all conditions. Mean maximum current amplitudes in the presence of $140 \mathrm{~mm}$ external $\mathrm{Na}^{+}$and 0.5 $\mathrm{mm}$ glutamate were $5.6 \pm 0.4 \mathrm{nA}(n=25)$ at $+165 \mathrm{mV}$ with standard $\mathrm{NaCl}$-based internal and standard $\mathrm{NaNO}_{3}$-based external solution and $-4.0 \pm 0.3 \mathrm{nA}(n=55)$ at $-175 \mathrm{mV}$ with standard $\mathrm{NaNO}_{3}$-based internal and standard $\mathrm{NaCl}$-based external solution. The mean maximum voltage error was calculated to be $2.6 \pm 0.2 \mathrm{mV}(n=80)$. Currents were filtered at $5 \mathrm{kHz}(-3 \mathrm{~dB})$ and digitized with a sampling rate of $50 \mathrm{kHz}$ using a Digidata (Molecular Devices) analog-to-digital converter. Cells were clamped to $0 \mathrm{mV}$ for at least $5 \mathrm{~s}$ between test sweeps. If not otherwise stated, standard solutions were used that contained the following (in mM): $140 \mathrm{NaNO}_{3}$ (standard $\mathrm{NaNO}_{3}$-based external solution) or $\mathrm{NaCl}$ (standard $\mathrm{NaCl}$-based external solution), $4 \mathrm{KCl}, 2 \mathrm{CaCl}_{2}, 1 \mathrm{MgCl}_{2}$, and 5 HEPES, pH 7.4. Intracellular solution contained the following (in $\mathrm{mm}$ ): $115 \mathrm{NaCl}$ (standard $\mathrm{NaCl}$-based internal solution) or $\mathrm{NaNO}_{3}$ (standard $\mathrm{NaNO}_{3}$-based external solution), $2 \mathrm{MgCl}_{2}, 5$ EGTA, and $10 \mathrm{HEPES}, \mathrm{pH}$ 7.4. For the concentration dependences of $\mathrm{Na}^{+}$and glutamate (see Fig. 2-4, 6), external $\mathrm{Na}^{+}$was substituted equimolarly with $\mathrm{K}^{+}$or a given concentration of L-glutamate was added to the external solution, respectively. For determination of external anion permeability ratios, cells were moved into a stream of solutions containing various sodium salts (in mM): $140 \mathrm{NaX}, 4 \mathrm{KCl}, 2 \mathrm{CaCl}_{2}, 1 \mathrm{MgCl}_{2}$, and 5 HEPES, pH 7.4 (X denotes $\mathrm{Cl}^{-}, \mathrm{NO}_{3}{ }^{-}$, and $\mathrm{SCN}^{-}$).

Data analysis. Data were analyzed with a combination of pClamp9 (Molecular Devices) and SigmaPlot (Jandel Scientific, San Rafael, CA) programs. Current amplitudes were used without applying a subtraction procedure. Current-voltage relationships at various substrate concentrations were constructed by plotting isochronal current amplitudes determined $1 \mathrm{~ms}$ after the voltage step versus the membrane potential. To obtain the concentration dependence of anion channel activation by $\mathrm{Na}^{+}$and glutamate, isochronal anion current amplitudes were measured at various concentrations at a given test potential. The so-obtained substrate dependences were fit with Hill equations

$$
I=\frac{I_{\max }[\text { substrate }]^{n}}{[\text { substrate }]^{n}+K_{\mathrm{D}}{ }^{n}}+I_{\mathrm{o}},
$$

and averaged after normalization to the maximum current amplitude $\left(I_{\max }+I_{\mathrm{o}}\right)$. The Hill coefficients $(n)$ were determined as fit parameter with the restriction to be an integer. The residual errors of these fits $(<0.01)$ were not significantly different from fits in which the Hill coef- ficient was allowed to assume non-integer values. In Figure 3, various Hill equations with given Hill parameters were fitted to the experimental values. For each membrane potential, apparent dissociation constants ( $K_{\mathrm{D}}$ values) were determined at several cells and averaged. The voltage dependence of the relative open probability (see Fig. 7, Table 1) was determined by plotting the normalized isochronal current amplitude at +135 or $-135 \mathrm{mV}$ after $0.2 \mathrm{~s}$ prepulses to different voltages versus the preceding potential. Activation curves obtained in this manner were then fit with a single Boltzmann term plus a voltage-independent value $\left[I(V)=\mathrm{Amp} /\left(1+e^{(V-V 0.5) / k V}\right)+P_{\min }\right]$. To determine the time course of current activation, deactivation, or inactivation (Table 1), a sum of two exponentials and a time-independent value $\left[I(t)=a_{1} \exp \left(-t / \tau_{\text {fast }}\right)+\right.$ $a_{2} \exp \left(-t / \tau_{\text {slow }}\right)+c$ ) were fit to data recorded during a series of voltage steps from a holding potential of $0 \mathrm{mV}$. Permeability ratios were calculated from reversal potential measurements using the GoldmanHodgkin-Katz equation (Hodgkin and Katz, 1949) as described previously (Melzer et al., 2003). The ratio of coupled and uncoupled transport (see Fig. 5D) was calculated by dividing the mean glutamate-induced current increases determined in the presence of internal $\mathrm{K}^{+}$by mean values of current increases in its absence determined in a different group of cells and by subsequent subtraction of 1 (see Results). For statistic evaluation, the Student's $t$ test was used. Insight II (Accelrys, Cambridge, UK) was used to generate the ribbon presentation shown in Figure $5 B$ and to determine distances between the $\alpha$-carbones of different residues.

Expression in Xenopus oocytes and radiotracer flux experiments. Uptake of L- $\left[{ }^{3} \mathrm{H}\right]$ glutamate (Amersham Biosciences, Freiburg, Germany) was measured in oocytes expressing WT, G464S, and Q467S EAAT4. WT and mutant rEAAT4 were subcloned into the pGEMHE vector (Liman et al., 1992) using EcoRI and HindIII sites. Capped cRNA was synthesized from NheI- or SpHI-linearized pGEMHE-rEAAT4 templates through use of the MESSAGE machine (Ambion, Austin, TX), resuspended in $10 \mu \mathrm{l}$ of water, and stored in $2 \mu \mathrm{l}$ aliquots at $-80^{\circ} \mathrm{C}$ until use. The cRNA was injected using a nanoliter injector (nanoliter 2000; World Precision Instruments, Sarasota, FL), and oocytes were kept at $18^{\circ} \mathrm{C}$ in ND-96 solution supplemented with $2.5 \mathrm{~mm}$ sodium pyruvate and $100 \mu \mathrm{g} / \mathrm{ml}$ gentamycin sulfate until recording (Neely et al., 2004). At 5 d after cRNA injection, oocytes were placed into a tube with $100 \mu \mathrm{ND}_{\mathrm{SCN}} 96$ buffer containing $96 \mathrm{~mm} \mathrm{NaSCN}, 4 \mathrm{~mm} \mathrm{KCl}, 1.3 \mathrm{~mm} \mathrm{CaCl}_{2}, 5 \mathrm{~mm}$ HEPES, and various concentrations of $\mathrm{L}-\left[{ }^{3} \mathrm{H}\right]$ glutamate and $\mathrm{L}$-glutamate in a ratio of 1:6000 above $300 \mu \mathrm{l}$ of mineral oil. After $30 \mathrm{~min}, \mathrm{~L}-\left[{ }^{3} \mathrm{H}\right]$ glutamate uptake was terminated by centrifuging the oocytes into the mineral oil layer. Uptake of L- $\left[{ }^{3} \mathrm{H}\right]$ glutamate was linear for at least $40 \mathrm{~min}$ (data not shown). Oocytes were transferred to $0.5 \%$ SDS and subjected to scintillation counting after lysis. For each tested experimental condition, uptake was also determined for at least two uninjected oocytes. These control values were averaged and subtracted from radioactive uptake levels measured on injected oocytes. Subtracted uptake values were normalized to values obtained with $100 \mu \mathrm{M}$ L-glutamate and averaged. The soobtained concentration dependences of glutamate uptake were fitted with Hill equations. The Hill coefficients were determined as fit parameter with the restriction to be an integer. In Figure $3 E$, Hill equations with given Hill parameters were fitted to the experimental values.

\section{Results}

\section{Transporter substrates increase the amplitudes and change the voltage dependence of EAAT4 anion currents}

We expressed EAAT4 glutamate transporters heterologously in tsA201 cells and measured currents through whole-cell patch clamping. Cells were intracellularly dialyzed with $\mathrm{K}^{+}$-free solutions to abolish the glutamate uptake current. The so-obtained whole-cell currents predominantly represent EAAT4 anion currents, because complete substitution with impermeant anions results in negligible current amplitudes [current amplitudes at $+165 \mathrm{mV}$ at $140 \mathrm{~mm}$ external $\mathrm{NaNO}_{3}$ and $115 \mathrm{~mm}$ internal $\mathrm{NaCl}$ with $0.5 \mathrm{~mm}$ glutamate, $5.6 \pm 0.4 \mathrm{nA}(n=25)$ compared with $0.2 \pm 0.04 \mathrm{nA}(n=5)$ after complete substitution of internal and external anions with gluconate in the presence of $0.5 \mathrm{~mm}$ glutamate] (Melzer et al., 2005). Figure 1 shows representative current 


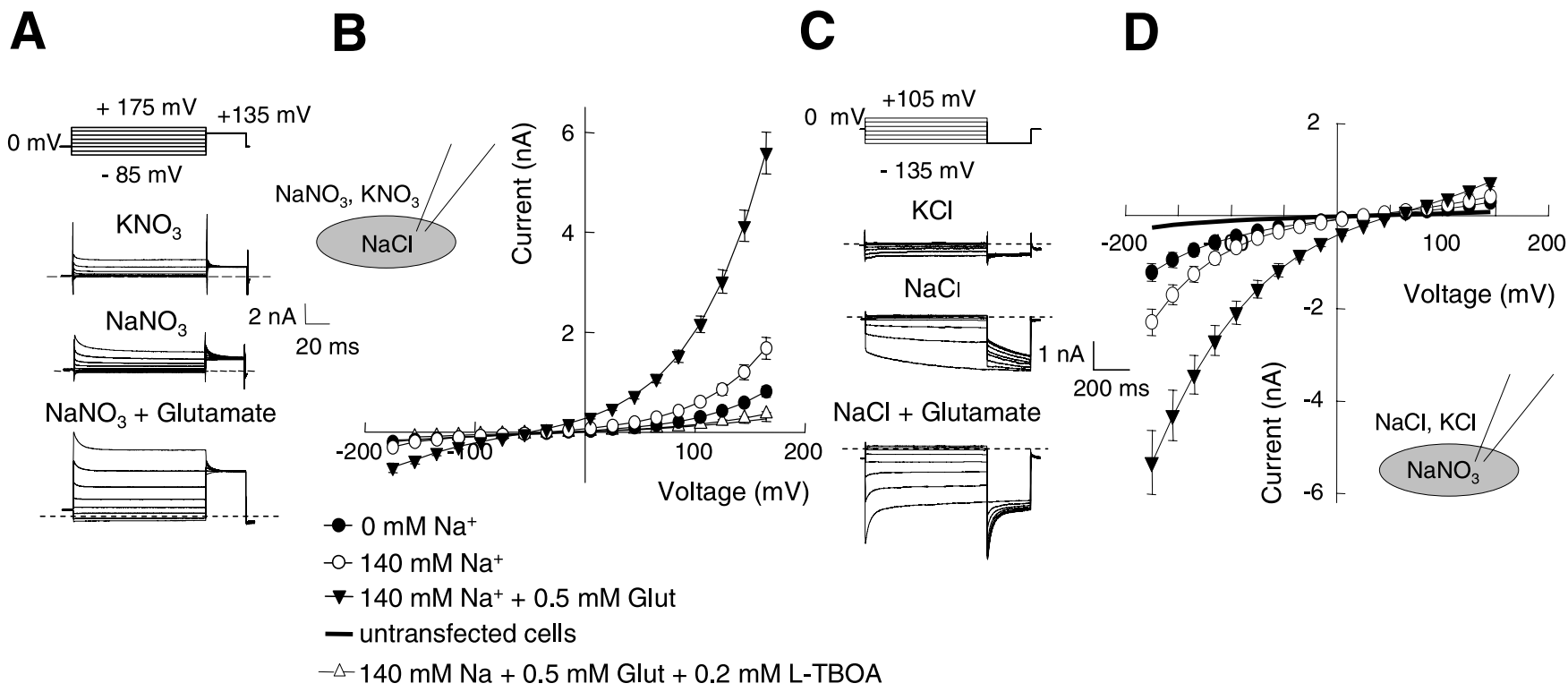

Figure 1. Activation of EAAT4 anion channels by transporter substrates. $\boldsymbol{A}, \boldsymbol{C}$, Pulse protocols and representative whole-cell current traces recorded from tsA201 cells expressing EAAT4 transporters. Cells were dialyzed with $\mathrm{NaCl}-(\boldsymbol{A})$ or $\mathrm{NaNO}_{3}-(\boldsymbol{C})$ based internal solutions and perfused with the indicated solutions. Dashed lines represent 0 current amplitude. $\boldsymbol{B}, \boldsymbol{D}$, Mean current-voltage relationships in the absence of $\mathrm{Na}^{+}$with $\mathrm{K}^{+}$as the main extracellular cation (filled circles; $n=5$ ), in the presence of $140 \mathrm{~mm} \mathrm{Na}^{+}$(open circles; $n=13$ in $\boldsymbol{B}$ and $n=12$ in $\boldsymbol{D}$ ), and in the presence of $140 \mathrm{~mm} \mathrm{Na}^{+}$and $0.5 \mathrm{~mm}$ glutamate (Glut; inverted filled triangles; $n=25$ in $B$ and $n=13$ in $\boldsymbol{D}$ ). Open triangles give mean current amplitudes from cells perfused with $0.2 \mathrm{mmL}$-TBOA ( $n=$ 5), and the solid line represent current amplitudes from untransfected tsA201 cells measured in $140 \mathrm{~mm} \mathrm{Na}{ }^{+}$-containing external solutions in the presence of $0.5 \mathrm{~mm}$ glutamate $(n=5)$.

responses and mean current amplitudes from transfected tsA201 cells at two different anion compositions. In Figure 1, $A$ and $B$, cells were internally dialyzed with an $\mathrm{NaCl}$-based solution and perfused with a solution containing $\mathrm{NO}_{3}{ }^{-}$constituting the main external anion. In Figure 1, $C$ and $D$, an inverted anion distribution with $\mathrm{NO}_{3}{ }^{-}$as main internal and $\mathrm{Cl}^{-}$as main external anion was used. To each cell, three external solutions were consecutively applied: a solution lacking $\mathrm{Na}^{+}$and glutamate with $\mathrm{K}^{+}$as main extracellular cation, a solution with a physiological external $\mathrm{Na}^{+}$concentration without glutamate, and one containing both glutamate and $\mathrm{Na}^{+}$. Currents were small in the absence of external $\mathrm{Na}^{+}$and markedly increased after the application of substrates (Fig. 1). For all tested ionic conditions, current amplitudes were significantly larger than in untransfected tsA201 cells (Fig. $1 B, D)$. Moreover, all currents can be completely blocked, i.e., to the levels of background currents, by $200 \mu \mathrm{M}$ L-threo- $\beta$ benzyloxyaspartate (L-TBOA) (Fig. $1 B$ ), a known blocker of EAAT4-associated anion channels (Shimamoto et al., 1998).

Cells were held at $0 \mathrm{mV}$, and voltage steps between -175 and $+165 \mathrm{mV}$ were applied. With $\mathrm{NO}_{3}{ }^{-}$on the extracellular membrane site, EAAT4-associated anion currents rise instantaneously during voltage steps to negative and positive potentials (Fig. 1A). At positive potentials, this instantaneous rise is followed by a time- and voltage-dependent current decay in the presence of sodium, as well as in the presence of both sodium and glutamate, but not in the absence of both substrates. Application of glutamate changes the time course of inactivation and makes steadystate inactivation less complete (Melzer et al., 2003). Gating of EAAT4 anion channels is completely different when studied with internal $\mathrm{NO}_{3}{ }^{-}$and external $\mathrm{Cl}^{-}$(Fig. 1C). In the absence of transporter substrates, currents are inwardly rectifying and time independent. $\mathrm{Na}^{+}$and glutamate increase the amplitude and alter the time and voltage dependence of EAAT4 anion currents. During application of an $\mathrm{Na}^{+}$-containing external solution lacking glutamate, voltage step to more negative voltages elicit a timedependent increase of current amplitudes. Depolarizing steps re- sult in time-independent current amplitudes (Fig. 1C). Application of glutamate causes an inversion of the activation curve of EAAT4 anion channels. Under these conditions, negative voltage steps elicit an instantaneous rise of the current amplitude followed by a time-dependent decrease (Fig. 1C).

\section{Concentration dependence of external $\mathrm{Na}^{+}$and glutamate}

We next measured EAAT4 anion currents at various concentrations of external $\mathrm{Na}^{+}$and glutamate (Figs. 2, 3). Isochronal current amplitudes determined at one cell at a fixed membrane potential under different $\left[\mathrm{Na}^{+}\right]$and [glutamate] were normalized, averaged, and plotted versus the ligand concentration. A comparison of isochronal current amplitudes at different times demonstrated that capacitive currents mediated by the transporter (Mim et al., 2005) do not modify the so-determined substrate dependences (data not shown). Figure $2 \mathrm{~A}$ shows the current responses to voltage steps between -175 and $+165 \mathrm{mV}$ of a single cell, consecutively exposed to standard $\mathrm{NO}_{3}{ }^{-}$-based and glutamate-containing external solutions with three different $\left[\mathrm{Na}^{+}\right]$of 0,20 , and $140 \mathrm{~mm}$. In all of these experiments, the external glutamate concentration was $0.5 \mathrm{~mm}$. Figure $2 B$ gives the sodium dependence of the isochronal EAAT4 anion current at a test potential of $+165 \mathrm{mV}$ obtained from such recordings. $\mathrm{Na}^{+}$ increases EAAT4 anion currents in sigmoidal relationship with a $K_{\mathrm{D}}$ of $31.6 \pm 2.6 \mathrm{~mm}(n>4)$ and a Hill coefficient of 3 . Apparent dissociation constants were determined for various other test potentials between +25 and $+165 \mathrm{mV}$ without appreciable voltage dependence (Fig. $2 \mathrm{~B}$, inset). In the absence of external $\mathrm{Na}^{+}$, the current amplitude is $\sim 20 \%$ of the maximum current amplitude. These currents are not contaminations by endogenous channels, because addition of L-TBOA reduces this anion current amplitude to $3.5 \%$ of the maximum current amplitude and because the relative current amplitude in the absence of $\mathrm{Na}^{+}$is independent of the EAAT4 expression level (data not shown). Similar results were obtained in experiments in which cells were dialyzed with $\mathrm{NaNO}_{3}$-based internal and $\mathrm{NaCl}$-based external solutions (Fig. 
A B

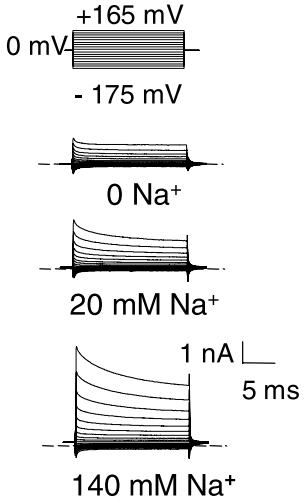

$140 \mathrm{mM} \mathrm{Na}^{+}$

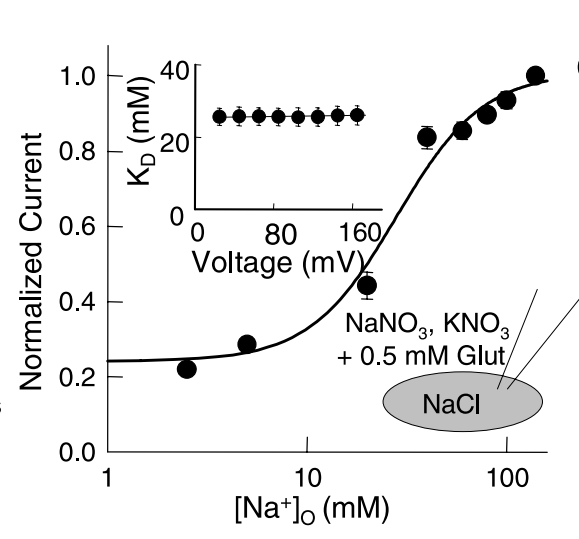

$\left[\mathrm{Na}^{+}\right]_{\mathrm{O}}(\mathrm{mM})$
C

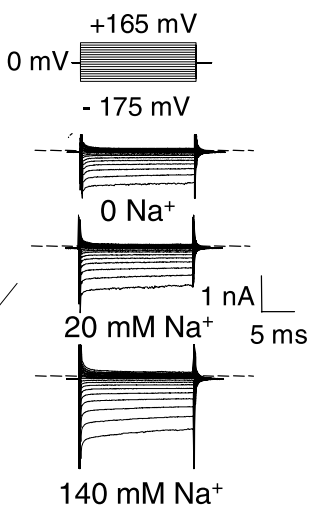

$140 \mathrm{mM} \mathrm{Na}^{+}$
D

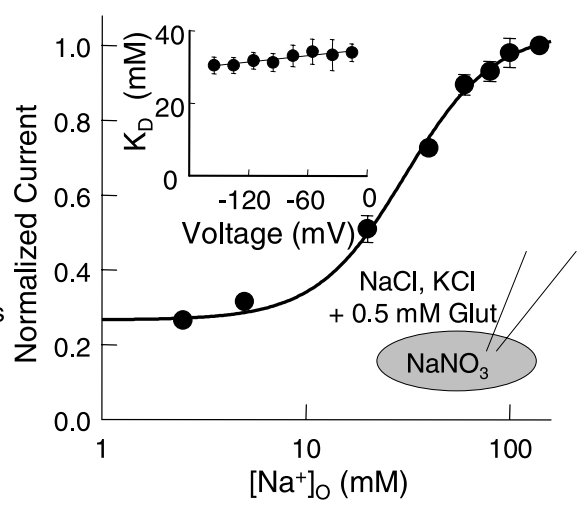

Figure 2. Sodium dependence of EAAT4 anion currents. $A, C$, Pulse protocols and representative whole-cell current traces recorded from tsA201 cells expressing EAAT4 transporters at different external sodium concentrations. Cells were dialyzed with either an $\mathrm{NaCl}_{-}(\boldsymbol{A})$ or $\mathrm{NaNO}_{3}-(\boldsymbol{C})$ based internal solution, respectively. $\boldsymbol{B}, \boldsymbol{D}$, Sodium dependences of isochronal anion current amplitudes measured at +165 and $-175 \mathrm{mV}$, respectively. Means \pm SEM; $n=6(\boldsymbol{B})$ and $n=5(\boldsymbol{D})$. The solid lines represent fits with the Hill equation with a coefficient of 3 . Insets, Plot of apparent dissociation constants ( $K_{D}$ values) for various voltages versus the test potentials. In all experiments, $0.5 \mathrm{~mm}$ glutamate (Glut) is present, and the extracellular [ $\mathrm{Na}^{+}$] was modified by equimolar substitution of $\mathrm{Na}^{+}$by $\mathrm{K}^{+}$.

$2 C, D)$. Figure 3 gives the glutamate dependence of anion currents with $\mathrm{NaCl}$-based internal and $\mathrm{NaNO}_{3}$-based external solution $(A, B)$ or $\mathrm{NaNO}_{3}$-based internal and $\mathrm{NaCl}$-based external solution $(C, D)$. Again, glutamate binds with voltage-independent $K_{\mathrm{D}}$ values of $35.0 \pm 0.3 \mu \mathrm{M}$ (with internal $\mathrm{Cl}^{-}$and external $\mathrm{NO}_{3}{ }^{-}$; $n=5$ ) and $51.0 \pm 3.0 \mu \mathrm{M}$ (with internal $\mathrm{NO}_{3}{ }^{-}$and external $\mathrm{Cl}^{-}$; $n>4 ; p>0.05$ ) (Fig. 3). At both anion distributions, there is a marked cooperativity in the glutamate dependence that results in a Hill coefficient of 3 when fitted with a Hill equation (Fig. $3 B, D$ ). EAAT4 anion channels are active in the absence of glutamate, conducting $\sim 20 \%$ of the maximum current amplitude. The glutamate dependence of anion currents were repeated with cells dialyzed with a $\mathrm{K}^{+}$-containing solution without change of apparent dissociation or Hill coefficient (data not shown). In contrast, substitution of external $\mathrm{NO}_{3}{ }^{-}$with $\mathrm{SCN}^{-}$caused an increase of the Hill coefficient from 3 to 5 (data not shown), demonstrating a role of the permeating anion in determining the cooperativity of the glutamate dependence.

Although the measured sodium dependence of anion currents is in agreement with the idea of multiple cotransported $\mathrm{Na}^{+}$ions (Fig. 2) (Watzke et al., 2001), the cooperativity of the glutamate dependence is at first glance surprising. EAAT2 and EAAT3 transport one glutamate together with three $\mathrm{Na}^{+}$, one $\mathrm{K}^{+}$, and one proton (Zerangue and Kavanaugh, 1996; Levy et al., 1998). The observed glutamate dependence of the EAAT4 anion current might be attributable to a different transport stoichiometry in this particular isoform or, alternatively, to a cooperative interaction of several carrier domains in regulating the anion current. To distinguish between these two possibilities, we determined the concentration dependence of EAAT4 glutamate uptake using radiotracer flux measurements (Fig. 3E). Xenopus oocytes were incubated in different glutamate concentrations at a fixed ratio of radioactive and nonradioactive glutamate, and the amount of transported $\mathrm{L}-\left[{ }^{3} \mathrm{H}\right]$ glutamate was determined after $30 \mathrm{~min}$. With an external solution containing $140 \mathrm{~mm} \mathrm{NaSCN}$, the anion current largely exceeds the glutamate uptake current (Melzer et al., 2003), clamping the oocyte membrane to the anion reversal potential. This procedure prevents membrane depolarization by electrogenic glutamate uptake and thus improves the accuracy in determining the concentration dependence of glutamate uptake. Figure $3 E$ gives the results of such experiments with different glutamate concentrations. Glutamate uptake increases with the extracellular glutamate concentration following a Hill equation, with a Hill coefficient of 1 and an apparent $K_{\mathrm{D}}$ of $8.5 \pm 0.8 \mu \mathrm{M}$. In contrast to the glutamate dependence of anion currents, there are no indications for cooperativity in glutamate uptake. We conclude from these experiments that only one glutamate is transported in each transport cycle of EAAT4 and that several cooperatively interacting glutamate binding sites need to be occupied to activate an EAAT4 anion channel.

Apparent dissociation constants and the cooperativity of glutamate and sodium dependences are additionally modified by the concentration of the other transporter substrate (Fig. 4). Figure $4 A$ gives the sodium dependence of the anion current for two different external glutamate concentrations ( 0.05 and $0.5 \mathrm{~mm}$ ). The cells were dialyzed with an $\mathrm{NaNO}_{3}$-based standard internal solution and externally perfused with an $\mathrm{NaCl}$-based external solution. A reduction of the external glutamate concentration leads to increased apparent dissociation constants of sodium binding, from $31.7 \pm 2.6 \mathrm{~mm}(0.5 \mathrm{~mm})$ to $67.6 \pm 1.4 \mathrm{~mm}(0.05$ $\mathrm{mm}$ ), without obvious change of cooperativity (Fig. $4 A$ ). For the glutamate dependence, a reduction of $\left[\mathrm{Na}^{+}\right]$from 140 to $40 \mathrm{mM}$ causes an increase of the $K_{\mathrm{D}}$ from $51.1 \pm 2.7$ to $206 \pm 20.7 \mu \mathrm{M}$ and a change of the Hill coefficient from 3 to 1 (Fig. $4 B$ ). Under all conditions, apparent dissociation constants are voltage independent (Fig. 4, insets).

\section{Point mutations affecting glutamate and $\mathrm{Na}^{+}$binding and anion channel gating}

The results presented so far suggest cooperativity between separate sodium and glutamate binding sites in the activation of the EAAT4 anion channels. We evaluated two mutant EAAT4 transporters, G464S and Q467S EAAT4, to study this phenomenon in more detail. Rat and human EAAT2 differ from all other mammalian EAAT isoforms in two serine residues at position 440 and 443 (in the EAAT2 numbering) (Fig. 5A) located between hairpin $2 \mathrm{~A}$ and $2 \mathrm{~B}$ in the structure of the Pyrococcus horikoshii glutamate transporter (Yernool et al., 2004) (Fig. 5B). These positions were 
A

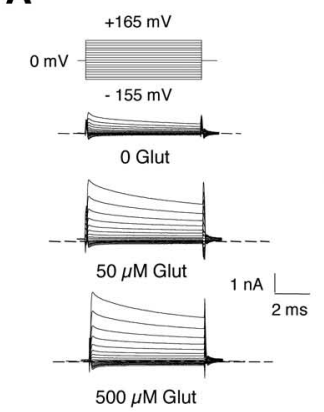

C

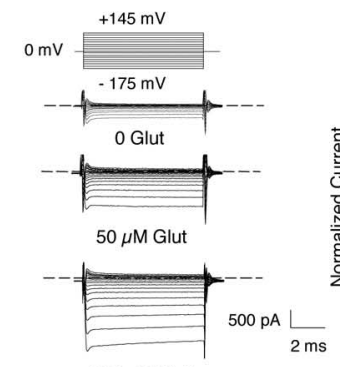

$500 \mu \mathrm{M}$ Glut

\section{E}

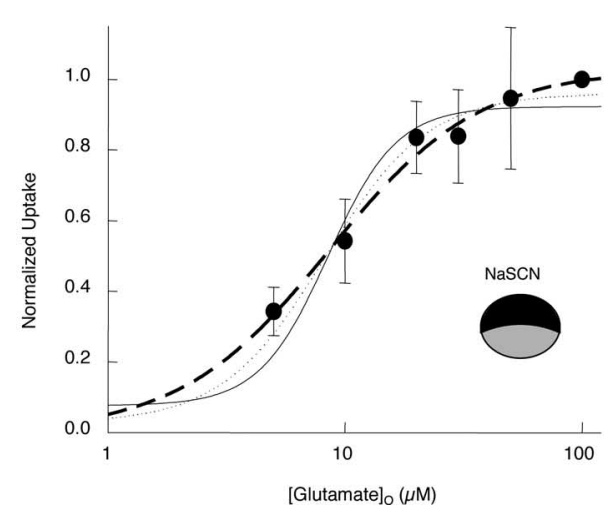

Figure 3. Glutamate dependence of EAAT4 anion currents and glutamate uptake. $A, C$, Pulse protocols and representative whole-cell current traces recorded from tsA201 cells expressing EAAT4 transporters at different glutamate (Glut) concentrations. Cells were dialyzed with either $115 \mathrm{~mm} \mathrm{NaCl}-(\boldsymbol{A})$ or $\mathrm{NaNO}_{3}$-( $\boldsymbol{C}$ based internal solutions and externally perfused with 140 $\mathrm{NaNO}_{3}$ - and $\mathrm{NaCl}$-based solutions, respectively. $\boldsymbol{B}, \boldsymbol{D}$, Glutamate dependence of anion currents for $\mathrm{NaCl}-(\boldsymbol{B})$ and $\mathrm{NaNO}_{3}$-based (D) internal solutions. Means \pm SEM; $n=5(\boldsymbol{B})$ and $n=8(\boldsymbol{D})$, respectively. Lines represent fits with the Hill equation with different fixed Hill coefficients $(n=$ 1 , dashed line; $n=2$, dotted line; and $n=3$, solid line). Insets give the voltage dependences of the apparent dissociation constants for glutamate ( $K_{D}$ values). $\boldsymbol{E}$, Glutamate dependence of the normalized radioactive glutamate uptake into oocytes expressing EAAT4 glutamate transporters in $96 \mathrm{~mm}$ NaSCN external-based solution. Means \pm SEM from eight oocytes. Fits with the Hill equation with different Hill coefficients are shown as dashed $(n=1)$, dotted $(n=2)$, and solid $(n=3)$ lines. In the experiments shown in $\boldsymbol{A}-\boldsymbol{D}$, the extracellular [ $\mathrm{Na}^{+}$] was $140 \mathrm{~mm}$ and in $\boldsymbol{E}$ was $96 \mathrm{~mm}$.

postulated to play a role in glutamate and sodium binding (Zhang and Kanner, 1999). Assuming that the three-dimensional structures of EAAT4 and the P. horikoshii glutamate transporter are comparable, these residues have distances of $\sim 20 \AA$ (G464) or $24 \AA$ (Q467) to the center of the protein and of $37 \AA$ (G464) or 41 $\AA$ (Q467) to the corresponding residue in neighboring subunits. We expressed G464S and Q467S EAAT4 transporters in Xenopus oocytes and tsA201 cells and studied radioactive glutamate uptake (Fig. 5) and anion currents (see Fig. 5-7) associated with mutant transporters.
A

$\mathbf{B}$
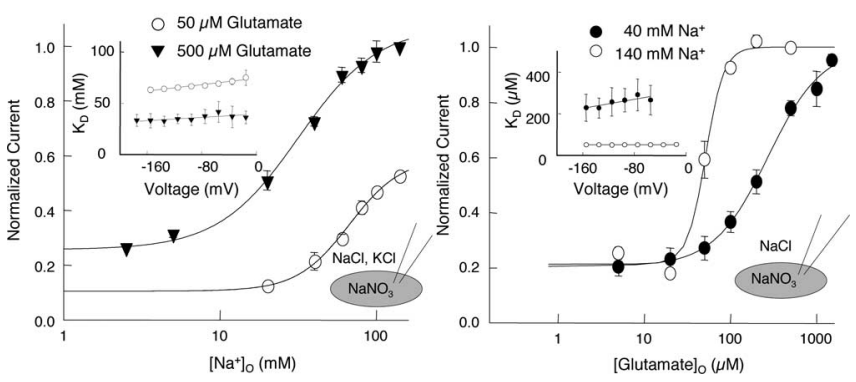

Figure 4. Sodium and glutamate interact in activating EAAT4 anion channels. $\boldsymbol{A}$, Sodium dependence of EAAT4 anion currents determined for two external glutamate concentrations (5 cells each). $\boldsymbol{B}$, Glutamate dependence of EAAT4 anion currents for two external sodium concentrations: $40 \mathrm{~mm} \mathrm{Na}^{+}$, filled circles $(n=6)$ and $140 \mathrm{~mm} \mathrm{Na}^{+}$, open circles $(n=8)$. Solid lines represent fits with Hill equations. Insets give the voltage dependence of apparent dissociation constants. Cells were internally dialyzed with $\mathrm{NaNO}_{3}$-based solution and externally perfused with $\mathrm{NaCl}$-based solution. The external $\left[\mathrm{Na}^{+}\right.$] was changed by equimolar substitution of $\mathrm{NaCl}$ by $\mathrm{KCl}$.

Absolute values as well as the glutamate dependence of L- $\left[{ }^{3} \mathrm{H}\right]$ glutamate uptake are comparable for WT and mutant EAAT4 $\left(K_{\mathrm{D}}\right.$ of $8.6 \pm 0.8 \mu \mathrm{M}$ for WT, $12.5 \pm 4.0 \mu \mathrm{M}$ for G464S, and $7.9 \pm 1.7 \mu \mathrm{M}$ for Q467S; $p>0.5$ ) (Fig. $5 C$ ). To calculate the ratios of coupled and uncoupled currents, glutamate-induced current increases were measured in transfected tsA201 cells internally dialyzed with a $\mathrm{K}^{+}$-containing solution and in cells with an internal $\mathrm{K}^{+}$-free solution, respectively. For these experiments, $\mathrm{Cl}^{-}$was chosen to be the main anion to increase the relative amplitude of the uptake current compared with the EAAT4 anion current. Because a lack of internal $\mathrm{K}^{+}$abolishes the coupled uptake current $\left(I_{\mathrm{GluNaHK}}\right)$ (Bergles et al., 2002) and because the chloride current in the absence of glutamate $\left(I_{\text {chloride }(-\mathrm{Glu})}\right)$ is independent of the internal cation (Melzer et al., 2003), one can calculate the ratio of uncoupled to coupled current by comparing relative current increases in the presence of internal $\mathrm{K}^{+}$divided by the corresponding values in its absence as follows:

$$
\begin{gathered}
\frac{\text { current increase }}{\text { current }} \frac{\left(\left[\mathrm{K}^{+}\right]_{\text {int }}=115 \mathrm{mM}\right)}{\left(\left[\mathrm{K}^{+}\right]_{\text {int }}=0\right)}=\frac{\frac{I_{\mathrm{GluNaHK}}+I_{\text {chloride }(+\mathrm{Glu})}}{I_{\text {chloride }(-\mathrm{Glu})}}}{\frac{I_{\text {chloride }(+\mathrm{Glu}))}}{I_{\text {chloride }(-\mathrm{Glu})}}} \\
=\frac{I_{\mathrm{GluNaHK}}+I_{\text {chloride }(+\mathrm{Glu})}}{I_{\text {chloride }(+\mathrm{Glu})}}=\frac{I_{\mathrm{GluNaHK}}}{I_{\text {chloride }(+\mathrm{Glu})}}+1
\end{gathered}
$$

Current increases were measured from cells dialyzed with a $\mathrm{K}^{+}$containing or a $\mathrm{K}^{+}$-free solution, respectively, mean values were calculated, and the ratio of the respective values was determined. This calculation reveals that, at $-175 \mathrm{mV}$ with $\mathrm{Cl}^{-}$as permeant anion, the glutamate uptake current measures $\sim 40 \%$ of the anion current in the presence of glutamate (Fig. 5). In agreement with the known voltage dependence of coupled glutamate transport (Zerangue and Kavanaugh, 1996), this analysis predicts a 0 glutamate uptake at $+125 \mathrm{mV}$. Neither G464S nor Q467S affect the so-obtained ratios of $I_{\mathrm{GluNaHK}}$ by $I_{\text {anion(+glu) }}($ at $-175 \mathrm{mV}$; WT, $0.40 \pm 0.01, n=8 ;$ G464S, $0.42 \pm 0.03, n=7$; Q467S, $0.45 \pm$ $0.03, n=7 ; p>0.1$ ) (Fig. $5 D$ ). Mutant transporters exhibit anion currents of comparable amplitude [without external glutamate at $+175 \mathrm{mV}$ with $\mathrm{Cl}_{\text {int }} / \mathrm{NO}_{3 \text { ext }}, 3.6 \pm 0.3 \mathrm{nA}$ for WT $(n=47), 3.6 \pm$ $0.3 \mathrm{nA}$ for G464S $(n=44)$, and $4.1 \pm 0.4 \mathrm{nA}$ for Q467S $(n=36)$; and at $-175 \mathrm{mV}$ with $\mathrm{NO}_{3 \text { int }} / \mathrm{Cl}_{\text {ext }},-2.6 \pm 0.2 \mathrm{nA}$ for WT $(n=$ 
A
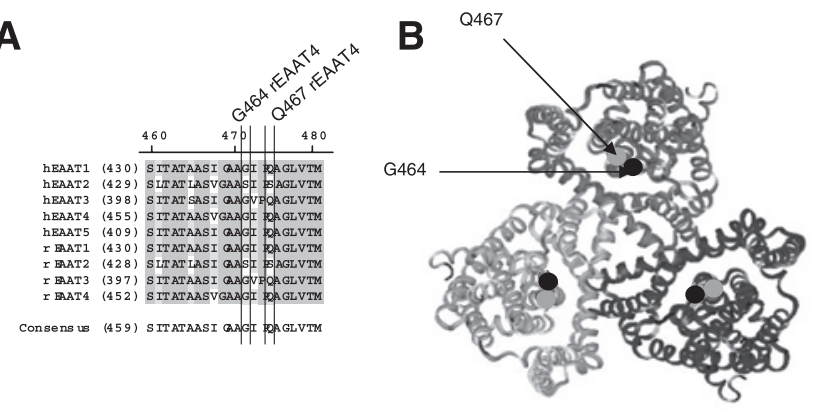

C

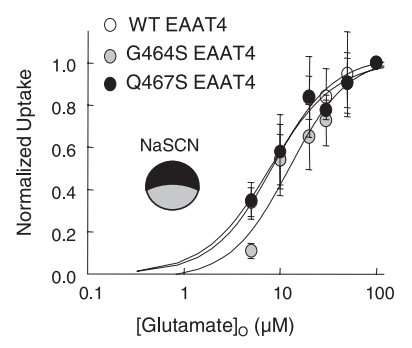

D

E

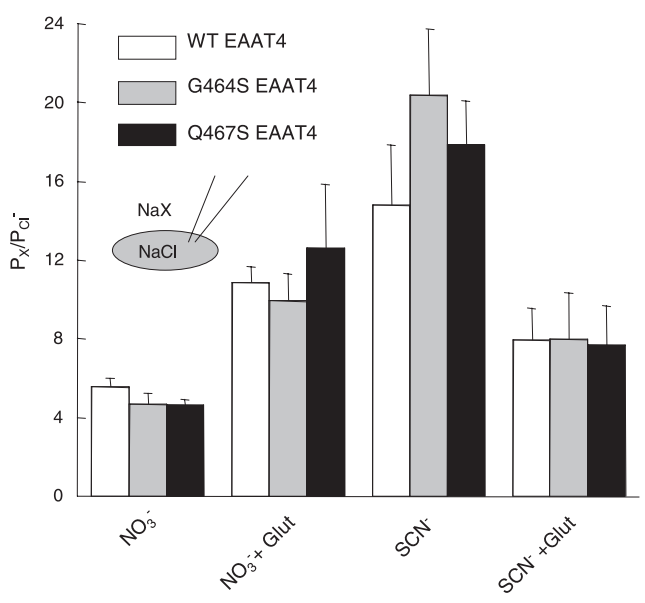

Figure 5. Effects of two point mutations close to the putative glutamate binding site on coupled and uncoupled transport. $\boldsymbol{A}$, Alignment of the region containing G464 and Q467 in various human and rat EAAT isoforms. $\boldsymbol{B}$, Localization of the residues corresponding to G464 (black) and Q467 (gray) of EAAT4 in the ribbon presentation of the three-dimensional structure of the P. horikoshii glutamate transporter (Yernool et al., 2004), viewed from the external membrane site. $C$, Glutamate dependence of radioactive glutamate uptake by WT and mutant EAAT4. Means \pm SEM from eight cells for each glutamate concentration. $D$, Ratio of uptake current to anion current amplitudes from at least five cells for WT and mutant EAAT4 transporters at $0.5 \mathrm{~mm}$ glutamate. $E$, Anion permeability ratios for WT and mutant EAAT 4 in the presence of $0.5 \mathrm{~mm}$ glutamate (Glut) for at least four cells.

$54),-2.1 \pm 0.3 \mathrm{nA}$ for G464S $(n=16)$, and $-2.4 \pm 0.3 \mathrm{nA}$ for Q467S $(n=16)$ ] and similar relative anion permeabilities (Fig. $5 E$ ). We conclude that the two mutations modify the amplitudes of neither coupled nor uncoupled transport.

The mutations do alter apparent dissociation constants and cooperativity of the glutamate and sodium concentration dependence of the anion current amplitude (Fig. 6). Figure 6 $\mathrm{A}$ shows the glutamate concentration dependence of WT, G464S, and Q467S EAAT4 anion currents. G464S causes an increase of the glutamate dissociation constant from $51.0 \pm 3.0$ to $183.5 \pm 3.4$ $\mu \mathrm{M}(n>4)$, whereas Q467S decreases the apparent dissociation constant to $41.3 \pm 3.4 \mu \mathrm{M}(n>3)$. Both mutations affect the cooperativity of glutamate binding. Whereas G464S increases the
A

B
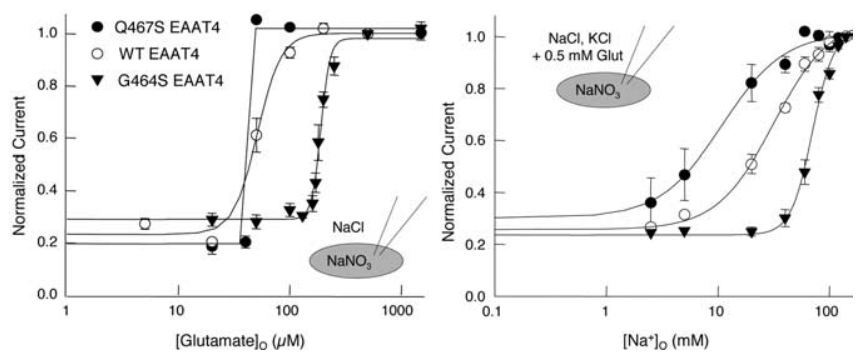

Figure 6. G464S and Q467S modify the intersubunit cooperativity in the EAAT4 anion channel activation. Plots of the normalized anion currents at $-155 \mathrm{mV}$ versus the glutamate (Glut) $(\boldsymbol{A})$ and the $\mathrm{Na}^{+}(\boldsymbol{B})$ concentration for WT, G464S, and Q467S EAAT4. Means \pm SEM from at least three $(\boldsymbol{A})$ or four $(\boldsymbol{B})$ cells, respectively. The cells were dialyzed with an $\mathrm{NaNO}_{3}$-based standard internal and perfused with an $\mathrm{NaCl}$-based standard external solution.

Hill coefficient from 3 to 15, the glutamate concentration dependence of Q467S EAAT4 is so steep that it cannot be satisfactorily fitted. In the presence of glutamate, G464S causes an increase of the apparent sodium dissociation constant from $31.7 \pm 2.6$ to $70.2 \pm 2.5 \mathrm{~mm}(n>3)$ and Q467S a decrease to $8.7 \pm 0.8 \mathrm{~mm}(n=$ 3 ). The sodium dependence of G464S fitted with a Hill equation gives a Hill coefficient of 4, in contrast to a Hill coefficient of 2 for Q467S and of 3 for WT EAAT4. All concentration dependences were identical for the two tested anion distributions (data not shown).

The two point mutations also alter anion channel gating (Fig. 7). So far, no coherent model of EAAT4 anion channel gating has been developed, and we therefore restrict the kinetic analysis to the experimentally observed time and voltage dependences of macroscopic currents (Table 1). At the two tested anion compositions, the mutations have distinct effects on channel gating. With external $\mathrm{NO}_{3}{ }^{-}$, WT and mutant channels inactivate during depolarizing voltage steps on a biexponential time course (Fig. 7A). Although deactivation time constants of G464S and Q467S EAAT4 anion channels are similar to WT without external glutamate, they are significantly increased in the presence of glutamate (Table 1). At the inverted anion distribution, WT and G464S EAAT4 anion channels activate during hyperpolarization in the absence of glutamate and deactivate at the same voltages in its presence. Activation was fit with a monoexponential and deactivation with a biexponential time course. The so-determined time constants are increased by G464S. For Q467S, activation in the absence of glutamate is basically absent (Fig. 7C), and there is no fast component of deactivation after application of glutamate. (Table 1). We next plotted instantaneous current amplitudes at a fixed test step versus the preceding potential (Fig. $7 B, D)$. These instantaneous current amplitudes depend on the number of anion channels per cell $(N)$, the unitary current amplitude $(i)$, and the absolute open probability at the end of the preceding voltage step $(p)(I=N i p)$. Assuming that no change in the open probability occurs during the voltage step to the test potential and that the unitary conductance is independent from the prepulse potential, the normalized instantaneous test step current amplitude is proportional to the absolute open probability at the end of the prepulse and thus gives the voltage dependence of the so-called relative open probability. The voltage dependence of the relative open probabilities of WT and mutant channels can be well described by a Boltzmann function for all constructs and conditions (Fig. 
A

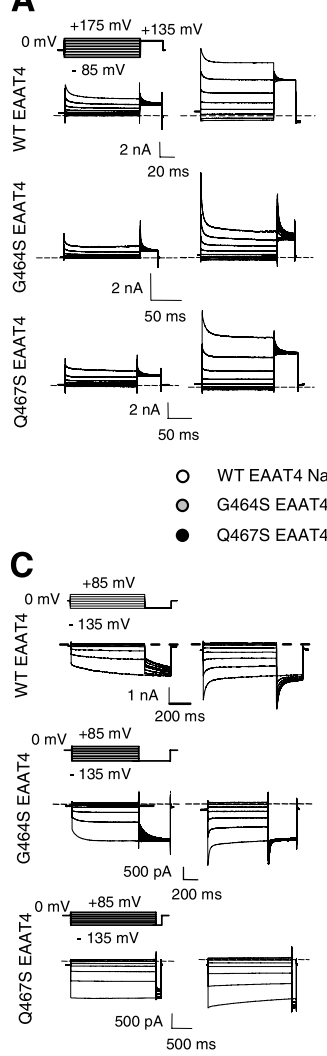

B

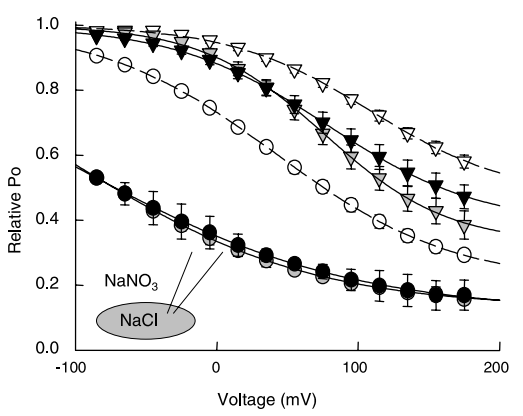

$\nabla \quad$ G464S EAAT4 $\mathrm{Na}^{+}+$Glut

$\checkmark$ Q467S EAAT4 $\mathrm{Na}^{+}+$Glut

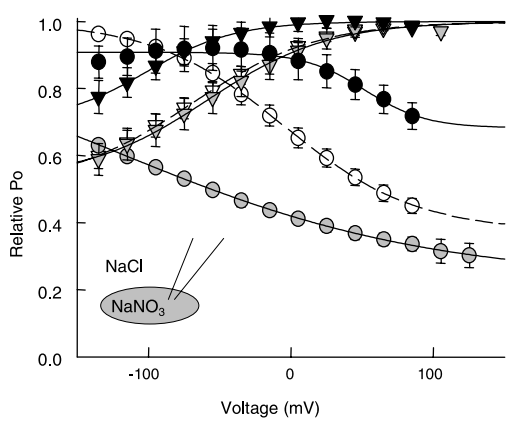

Figure 7. G464S and Q467S modify gating of EAAT4 anion channels. Representative current recordings for WT and mutant transporters for external $(\boldsymbol{A})$ or internal $(\boldsymbol{C}) \mathrm{NO}_{3}{ }^{-}$. Recordings in the left column were done in the absence of glutamate and those in the right column after applying $0.5 \mathrm{~mm}$ L-glutamate (Glut). $\boldsymbol{B}, \boldsymbol{D}$, Voltage dependence of the relative open probability for WT, G464S, and Q467S EAAT4 at the same anion conditions as in the corresponding left panel. Means \pm SEM from at least six cells $(\boldsymbol{B}, \boldsymbol{D})$.

$7 \mathrm{~B}, \mathrm{D})$. With $\mathrm{NO}_{3}{ }^{-}$on the external membrane site, both mutations shift the voltage dependence of inactivation to more negative potentials in both the presence and absence of glutamate. With internal $\mathrm{NO}_{3}{ }^{-}, \mathrm{G} 464 \mathrm{~S}$ shifts the activation curve determined without glutamate to more negative voltages, leaving the voltage dependence of activation in the presence of glutamate unaffected. Q467S increases the minimum open probability and shifts the voltage dependence of activation to more negative potential in the presence of glutamate. Without glutamate, the activation curve of Q467S EAAT4 anion channels has its midpoint at more positive potentials than WT channels. Our results demonstrate that mutations affecting binding of $\mathrm{Na}^{+}$and glutamate modify voltage- and aniondependent gating processes in EAAT4 anion channels.

\section{Mixed heterotrimers of WT and mutant EAAT4 with altered substrate selectivity}

The results presented so far indicate allosteric interactions between glutamate binding sites. Because each subunit is thought to function as independent glutamate transporter (Grewer et al., 2005; Koch and Larsson, 2005), our data support the concept of the three subunits cooperating in activating anion channels. This interpretation is in disagreement with a recent report by Grewer et al. (2005) who studied anion currents of mixed EAAT3 heterotrimers consisting of WT and a mutant with changed substrate selectivity (R446Q). In these experiments, the amplitudes of anion currents elicited at $0 \mathrm{mV}$

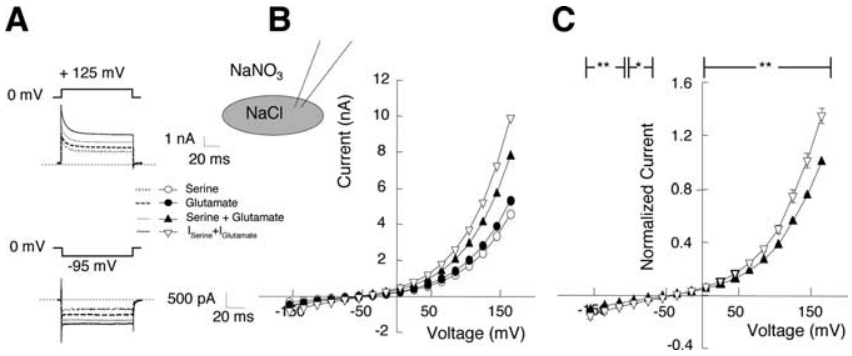

Figure 8. Mixed heterotrimers consisting of WT and R501C EAAT4 exhibit functional properties that are intermediate to WT and R501C homotrimers. $A$, Representative current responses to voltage steps to -95 and $+125 \mathrm{mV}$, respectively, from a cell cotransfected with WT and R501C EAAT4 in the presence of $1 \mathrm{~mm}$ serine (dotted line), $1 \mathrm{~mm}$ glutamate (dashed line), and both together (solid line). The bold solid line gives the sum of the current amplitudes observed after application of individual substrates. $\boldsymbol{B}$, Voltage dependence of current amplitudes from the experiment shown in $\boldsymbol{A}$. $\boldsymbol{C}$, Voltage dependence of normalized mean current amplitudes during the application of both substrates (filled triangles) compared with the predicted value assuming independence of individual subunits forming an anion conduction pathway (open inverted triangles). Means \pm SEM from 10 cells. ${ }^{*} p<0.05$ and ${ }^{* *} p<0.01$ indicate a significant difference between measured and predicted values.

by the mixture of two substrates were identical to the sum of the anion current amplitudes activated by the application of individual substrates. The authors concluded that each subunit forms an independently functioning anion conduction pathway.

To resolve this difference, we performed analogous experiments with EAAT4 (Fig. 8). We constructed a mutant EAAT4 plasmid that causes the substitution of arginine by cysteine at position 501 (corresponding to position 446 in rEAAT3). R501C EAAT4 expressed in tsA201 cells exhibit anion currents that are activated by serine but not by glutamate (supplemental Fig. 1, available at www.jneurosci.org as supplemental material), in agreement with results on similar EAAT3 mutants (Bendahan et al., 2000). If individual subunits formed separate and independent anion channels, one would expect additive effects of serine and glutamate on anion currents in cells coexpressing WT and R501C EAAT4. Figure 8 shows a representative recording and current-voltage relationships from such an experiment. The cell was held at $0 \mathrm{mV}$, and current responses to voltages between -155 and $+165 \mathrm{mV}$ were determined in the presence of $1 \mathrm{~mm}$ serine, $1 \mathrm{~mm}$ glutamate, or both substrates, respectively. Figure $8 B$ gives the voltage dependences of the current amplitudes measured at these conditions, together with the voltage dependence of the sum of the glutamate- and serine-induced current amplitudes. In this experiment, serine and glutamate do not exert additive effects. A comparison of mean values from 10 cells revealed that anion amplitudes in the presence of serine and glutamate were significantly smaller than the sum of the current amplitudes with only one substrate at all positive test potentials and at all potentials negative to $-50 \mathrm{mV}$ (Fig. $8 \mathrm{C}$ ). Hence, our experiments do not support the notion of a functional independence of EAAT4 subunits in activating anion currents.

\section{Discussion}

EAAT4 anion channels are active in the absence of glutamate (Fig. 1), and extracellular glutamate increases anion current amplitudes with a sigmoidal concentration dependence (Fig. 3). Fitting Hill equations result in Hill coefficients larger than 1, pointing toward the existence of more than one binding site and to a cooperativity between these multiple sites. Like EAAT2 and 
EAAT3, EAAT4 appears to transport only one glutamate together with $\mathrm{Na}^{+}, \mathrm{K}^{+}$, and proton (Zerangue and Kavanaugh, 1996; Levy et al., 1998), indicating that each carrier domain exhibits only a single glutamate binding site. Assuming that each subunit transports glutamate by itself (Grewer et al., 2005; Koch and Larsson, 2005), the cooperativity in glutamate binding in activating the anion channel must result from interactions between multiple subunits.

So far, the cooperativity in glutamate activation in EAAT-associated anion channels has not been reported. The deviation of our results from previous publications most likely arises from experimental differences. Glutamate dependences of EAAT4 currents on injected Xenopus oocytes (Lin et al., 1998; Mitrovic et al., 2001) were well fit with a Hill coefficient of 1 . In these experiments, transporters are exposed to a physiologically high internal $\left[\mathrm{K}^{+}\right]$and only $30 \mathrm{mM} \mathrm{Cl}^{-}$as only permeant internal anions (Cooper and Fong, 2001), likely causing an insufficient separation of anion and uptake currents. At negative voltages, relative anion current amplitudes are significantly smaller in Xenopus oocytes than in dialyzed mammalian cells because of the lower intracellular chloride concentration, and the uptake current amplitude will dominate the substrate dependence at this experimental condition. In exact agreement with this explanation, glutamate dependences of EAAT4 currents in Xenopus oocytes are less steep at negative than at positive potentials (data not shown). Otis and Jahr (1998) determined the glutamate dependence of native EAAT4 transporter-associated currents from Purkinje neurons by measuring peak current amplitudes at $-80 \mathrm{mV}$ after fast application of glutamate. Because EAAT4 anion currents exhibit voltage- and glutamate-dependent gating transitions (Melzer et al., 2003), such glutamate dependences also reflect gating transitions of this channel, which likely affect the steepness of the glutamate dependence. Another publication (Mim et al., 2005) reported experiments on EAAT4 transporters heterologously expressed in mammalian cells using whole-cell patch clamp. After subtraction of the current amplitudes in the absence of glutamate, the glutamate dependence measured on cells dialyzed with an $\mathrm{SCN}^{-}$containing solution at $0 \mathrm{mV}$ could be well fit with a Hill relationship and a Hill coefficient of 1 . Glutamate modifies the relative anion selectivity of the EAAT4 anion channel (Melzer et al., 2003), and this will affect current measurements at potentials close to the reversal potential. Moreover, EAAT4 expression levels in this series of experiments are much lower than ours. Current amplitudes at $0 \mathrm{mV}$ are therefore small and potentially affected by the glutamate-induced change of the reversal potentials at asymmetrical anion conditions.

Two tested point mutations, G464S and Q467S, modify the steepness of the glutamate dependence of the anion current amplitude, although both are far away from the center of the protein and the corresponding residue in neighboring subunits (Fig. 5B). The effects of these amino acid substitutions thus indicate that conformational changes of one subunit are sensed by its neighbors. Despite the pronounced effects on EAAT4 anion channels, G464S and Q467S have no effect on glutamate transport (Fig. 5), in agreement with the notion that glutamate transport occurs without intersubunit interactions (Grewer et al., 2005; Koch and Larsson, 2005).

Additional support for the idea of intersubunit interactions in EAAT anion transport comes from the finding that, in mixed heterotrimers consisting of WT and mutant EAAT4 with modified substrate selectivity, WT and mutant subunits do not conduct anions independently (Fig. 8). Our results differ from those reported by Grewer et al. (2005) who performed similar experiments with the result that different substrates have additive effects on heterotrimers out of WT and mutant EAAT3. Isoformspecific differences between EAAT3 and EAAT4 might account for this discrepancy. Because EAAT4 anion current activation is very complex, two substrates might have an additive effect on transporter currents under certain conditions in cotransfection experiments, although there are interactions between subunits. In contrast, every deviation from independence indicates that heterotrimeric transporters exhibit a novel substrate sensitivity attributable to cooperative interactions between subunits.

The EAAT-associated anion channel has been conceived as a glutamate-gated channel with a tight coupling of channel opening and closing to conformational changes of the glutamate carrier domain (Fairman et al., 1995; Wadiche et al., 1995; Billups et al., 1996; Larsson et al., 1996; Arriza et al., 1997; Wadiche and Kavanaugh, 1998; Watzke et al., 2001). In this model, only certain carrier conformations are associated with conducting anion pores. During transitions through various conformational states of the glutamate carrier the anion channel is thought to cycle between conducting and nonconducting states. A recent study on EAAT3-associated anion channels (Ryan et al., 2004), which demonstrated that many residues involved in anion selectivity are in close spatial proximity to regions presumed to play a role in substrate binding, supported this view. The finding that engineered cysteines at these positions form intrasubunit but not intersubunit disulfide bridges (Ryan et al., 2004) suggested that anion conduction pathways are formed by individual EAAT subunits that switch between glutamate transporting and anion conducting states. 
The here reported interactions between neighboring subunits that occur during activation of the EAAT4 anion channel, but not during glutamate transport, argue against this model. Moreover, the anion and voltage dependence of EAAT4 anion currents, shown here and in a previous report (Melzer et al., 2003), is inconsistent with a strict coupling of glutamate transport and anion channel activation. We did not see any decrease of the instantaneous or the steady-state anion current amplitudes up to an external glutamate concentration of $2 \mathrm{~mm}$ (data not shown). Time- and voltage-dependent current relaxations are observed in the absence of glutamate as well as at all glutamate concentrations. With internal $\mathrm{NO}_{3}{ }^{-}$, hyperpolarizing voltage steps cause a current increase at [glutamate] of $0 \mathrm{~mm}$ and a current decrease at [glutamate] of 0.5 mM. With external $\mathrm{NO}_{3}{ }^{-}$, depolarizing pulse causes a current decay. These results demonstrate that the voltage-dependent relaxations of EAAT4 anion currents are not caused by voltage-dependent glutamate binding, resulting in changed percentages of EAAT4 transporters binding glutamate, but rather by a voltage-dependent gating process.

Gating of EAAT4 anion channel depends on the identities and concentrations of permeant anions (Fig. 1). Inversion of the anion gradient across the membrane does not modify binding of $\mathrm{Na}^{+}$and glutamate (Figs. 2, 3). Hence, there must exist conformational changes of the anion channel that are not related to transitions between separate carrier states. EAAT4 anion channels can open and close in the absence of conformational changes of the glutamate carrier. The dependence of the anion distribution on the EAAT4 current relaxation is much more pronounced than for other channels (Marchais and Marty, 1979; Pusch et al., 1995; Chen and Miller, 1996). Although the open probability of the channels increases with more positive potentials in the presence of internal $\mathrm{NO}_{3}{ }^{-}$and external glutamate (Fig. $1 C$ ), in all other tested conditions channels deactivate during depolarization (Fig. 1A) or activate during hyperpolarization (Fig. 1C). One mutation, Q467S, alters the time course of anion currents in cells intracellularly dialyzed with the more permeant anion but affect channel gating only minimally under the inverse anion gradient (Fig. 7). A possible explanation for all of these finding is that EAAT4 anion channels are gated by permeant anions from both sites of the membrane. Glutamate and two mutations close to the glutamate binding pocket might alter EAAT anion channel gating via modification of binding and translocation of permeant anions (Melzer et al., 2003). By such a mechanism, EAAT4 anion channel gating, albeit not tightly coupled to transition within the carrier, is affected by conformational changes of the carrier.

Because glutamate is cotransported with multiple sodium ions (Zerangue and Kavanaugh, 1996; Levy et al., 1998), the observed sigmoidicity of the $\mathrm{Na}^{+}$dependence of the EAAT4 anion currents (Fig. 2) could be explained by an interaction between $\mathrm{Na}^{+}$binding steps within a single subunit (Watzke et al., 2001). However, the clear alterations of the sodium dependence of EAAT4 anion currents by the two tested mutations (Fig. 6B) together with the absent changes of coupled glutamate transport by $\mathrm{G} 464 \mathrm{~S}$ and Q467S EAAT4 transporters (Fig. 5C,D) suggest that intersubunit interactions also play a role in the sodium dependence of anion currents. Such intersubunit interactions also account for the observed modification of sodium and glutamate binding by the concentration of the other substrate (Fig. 4).

In conclusion, our data demonstrate that the three subunits of EAAT4 transporters cooperate in activating anion channels and individually mediate coupled glutamate transport. This finding suggests that either three anion channels, each formed by a single subunit, allosterically interact or more than one subunit contributes to the formation of the anion channels. Our results illustrate the importance of multimerization of this class of transporters and provide a likely explanation as to how a single protein can perform two mechanically distinct transport processes simultaneously.

\section{References}

Amara SG, Fontana AC (2002) Excitatory amino acid transporters: keeping up with glutamate. Neurochem Int 41:313-318.

Arriza JL, Eliasof S, Kavanaugh MP, Amara SG (1997) Excitatory amino acid transporter 5 , a retinal glutamate transporter coupled to a chloride conductance. Proc Natl Acad Sci USA 94:4155-4160.

Bendahan A, Armon A, Madani N, Kavanaugh MP, Kanner BI (2000) Arginine 447 plays a pivotal role in substrate interactions in a neuronal glutamate transporter. J Biol Chem 275:37436-37442.

Bergles DE, Tzingounis AV, Jahr CE (2002) Comparison of coupled and uncoupled currents during glutamate uptake by GLT-1 transporters. J Neurosci 22:10153-10162.

Billups B, Rossi D, Attwell D (1996) Anion conductance behavior of the glutamate uptake carrier in salamander retinal glial cells. J Neurosci 16:6722-6731

Chen TY, Miller C (1996) Nonequilibrium gating and voltage dependence of the ClC-0 Cl- channel. J Gen Physiol 108:237-250.

Cooper GJ, Fong P (2001) Relationship between intracellular pH and chloride in Xenopus oocytes expressing the chloride channel ClC-0. Am J Physiol Cell Physiol 284:C331-C338.

Eskandari S, Kreman M, Kavanaugh MP, Wright EM, Zampighi GA (2000) Pentameric assembly of a neuronal glutamate transporter. Proc Natl Acad Sci USA 97:8641-8646.

Fairman WA, Vandenberg RJ, Arriza JL, Kavanaugh MP, Amara SG (1995) An excitatory amino-acid transporter with properties of a ligand-gated chloride channel. Nature 375:599-603.

Gendreau S, Voswinkel S, Torres-Salazar D, Lang N, Heidtmann H, DetroDassen S, Schmalzing G, Hidalgo P, Fahlke C (2004) A trimeric quaternary structure is conserved in bacterial and human glutamate transporters. J Biol Chem 279:39505-39512.

Grewer C, Balani P, Weidenfeller C, Bartusel T, Tao Z, Rauen T (2005) Individual subunits of the glutamate transporter EAAC1 homotrimer function independently of each other. Biochemistry 44:11913-11923.

Haugeto O, Ullensvang K, Levy LM, Chaudhry FA, Honore T, Nielsen M, Lehre KP, Danbolt NC (1996) Brain glutamate transporter proteins form homomultimers. J Biol Chem 271:27715-27722.

Hodgkin AL, Katz B (1949) The effect of sodium ions on the electrical activity of the giant squid axon. J Physiol (Lond) 108:37-77.

Koch HP, Larsson HP (2005) Small-scale molecular motions accomplish glutamate uptake in human glutamate transporters. J Neurosci 25:1730-1736

Larsson HP, Picaud SA, Werblin FS, Lecar H (1996) Noise analysis of the glutamate-activated current in photoreceptors. Biophys J 70:733-742.

Levy LM, Warr O, Attwell D (1998) Stoichiometry of the glial glutamate transporter GLT-1 expressed inducibly in a Chinese hamster ovary cell line selected for low endogenous $\mathrm{Na}^{+}$-dependent glutamate uptake. J Neurosci 18:9620-9628.

Liman ER, Tytgat J, Hess P (1992) Subunit stoichiometry of a mammalian $\mathrm{K}^{+}$channel determined by construction of multimeric cDNAs. Neuron 9:861-871.

Lin CL, Tzingounis AV, Jin L, Furuta A, Kavanaugh MP, Rothstein JD (1998) Molecular cloning and expression of the rat EAAT4 glutamate transporter subtype. Brain Res Mol Brain Res 63:174-179.

Marchais D, Marty A (1979) Interaction of permeant ions with channels activated by acetylcholine in Aplysia neurones. J Physiol (Lond) 297:9-45.

Melzer N, Biela A, Fahlke C (2003) Glutamate modifies ion conduction and voltage-dependent gating of excitatory amino acid transporter-associated anion channels. J Biol Chem 278:50112-50119.

Melzer N, Torres-Salazar D, Fahlke C (2005) A dynamic switch between inhibitory and excitatory currents in a neuronal glutamate transporter. Proc Natl Acad Sci USA 102:19215-19218. 
Mim C, Balani P, Rauen T, Grewer C (2005) The glutamate transporter subtypes EAAT4 and EAATs 1-3 transport glutamate with dramatically different kinetics and voltage dependence but share a common uptake mechanism. J Gen Physiol 126:571-589.

Mitrovic AD, Plesko F, Vandenberg RJ (2001) $\mathrm{Zn}^{2+}$ inhibits the anion conductance of the glutamate transporter EAAT4. J Biol Chem 276:26071-26076.

Neely A, Garcia-Olivares J, Voswinkel S, Horstkott H, Hidalgo P (2004) Folding of active calcium channel $\beta 1 \mathrm{~b}$-subunit by size-exclusion chromatography and its role on channel function. J Biol Chem 279:21689-21694.

Otis TS, Jahr CE (1998) Anion currents and predicted glutamate flux through a neuronal glutamate transporter. J Neurosci 18:7099-7110.

Pusch M, Ludewig U, Rehfeldt A, Jentsch TJ (1995) Gating of the voltagedependent chloride channel $\mathrm{ClC}-0$ by the permeant anion. Nature 373:527-530.

Ryan RM, Mitrovic AD, Vandenberg RJ (2004) The chloride permeation pathway of a glutamate transporter and its proximity to the glutamate translocation pathway. J Biol Chem 279:20742-20751.

Shimamoto K, Lebrun B, Yasuda-Kamatani Y, Sakaitani M, Shigeri Y, Yu- moto N, Nakajima T (1998) DL-threo-beta-benzyloxyaspartate, a potent blocker of excitatory amino acid transporters. Mol Pharmacol 53:195-201.

Wadiche JI, Kavanaugh MP (1998) Macroscopic and microscopic properties of a cloned glutamate transporter/chloride channel. J Neurosci 18:7650-7661.

Wadiche JI, Amara SG, Kavanaugh MP (1995) Ion fluxes associated with excitatory amino acid transport. Neuron 15:721-728.

Watzke N, Bamberg E, Grewer C (2001) Early intermediates in the transport cycle of the neuronal excitatory amino acid carrier EAAC1. J Gen Physiol 117:547-562.

Yernool D, Boudker O, Jin Y, Gouaux E (2004) Structure of a glutamate transporter homologue from Pyrococcus horikoshii. Nature 431:811-818.

Zerangue N, Kavanaugh MP (1996) Flux coupling in a neuronal glutamate transporter. Nature 383:634-637.

Zhang Y, Kanner BI (1999) Two serine residues of the glutamate transporter GLT-1 are crucial for coupling the fluxes of sodium and the neurotransmitter. Proc Natl Acad Sci USA 96:1710-1715. 